\title{
PREVALENCE OF ANAEMIA IN PREGNANCY IN HOSPITAL BASED POPULATION IN BIJAPUR, KARNATAKA.
}

Mohammed Arifulla K ${ }^{1}$, A.M. Patil2 ${ }^{2}$ B. B. Sajjanar, Sayeed M Yendigeri ${ }^{3}$, Vivek P. Honakeri ${ }^{4}$, Nasheen Fathima 5 , Nida Nausheen 6

\section{HOW TO CITE THIS ARTICLE:}

Mohammed Arifulla K, AM Patil, B. B. Sajjanar, Sayeed M. Yendigeri, Vivek P. Honakeri, Nasheen Fathima, Nida Nausheen. "Prevalence of anaemia in pregnancy in hospital based population in Bijapur, Karnataka". Journal of Evolution of Medical and Dental Sciences 2013; Vol2, Issue 35, September 2; Page: 6673-6682.

ABSTRACT: BACKGROUND: Anaemia is a major public health problem during the pregnancy throughout the world. Globally almost half of the pregnant mothers are anemic. The hematological data representative of anaemia among Asian countries, India has highest prevalence and continues to be serious health problem. Studying the prevalence of Anemia in pregnancy aids in establishing the magnitude of the problem and helps the antenatal health providers in early detection and management. In a teaching hospital based a population study of Anemia in pregnancy is strong indication of true Anemia prevalence in the community, as large numbers of patients from different backgrounds attend the hospital. OBJECTIVES: The study was conducted to know the prevalence of Anemia in pregnancy in and around Bijapur, Karnataka. This study related with the Prevalence of Anemia in pregnancy in relation to age, gravida, parity, trimester and morphological types of Anemia. MATERIAL AND METHODS: The material for this study is obtained from in-patient and out-patients attending the OBG department at Al-Ameen Women's and children's hospital, Bijapur Automated hematology analyzer - Sysmex-Kx21 and peripheral smear study were used to study the anaemia in pregnancy. 100 blood samples of those pregnant women whose hemoglobin was less than $11 \mathrm{gm} / \mathrm{dl}$ were selected for study over a period of one year from January, 2012 to December 2012. The study was according to predesigned and Prescheduled proforma which includes detailed obstetrics history and systemic examination. LIMITATIONS OF STUDY: Serum iron, Serum Folic Acid, Serum ferritin, and Vitamin B12 assays, total Iron Binding Capacity (TIBC), Zinc, were not done because they are costly and most of patients were from poor socioeconomic backgrounds. RESULTS: Blood samples of 100 pregnant women were analyzed \& were found to be anaemic. This study includes magnitude of anaemia according to age, gravida and parity wise distribution of anaemia. degree, types of anaemia. Out of 100 pregnant cases, 70 had moderate degree, 12 cases had severe degree and 18 were mild degree anemia' Microcytic hypochromic were - 70 (70\%), Macrocytic Normochromic - 17 (17 \%) and dimorphic anaemia- 13 (13\%).

KEYWORDS: Anaemia, Pregnancy, prevalence, severity, grade, parity, and lower socio economic status, India and preventive.

INTRODUCTION: Anaemia is a global health problem. In Anemia, the lower limit of normality during the pregnancy is reduced. (1). In the pregnancy, anemia accounts for $40-70$ percent cases with the increasing incidence of parity(2), Anemia is the most common nutritional deficiency disorder, \& prevalence of anaemia in pregnancy is 51 percent in developing countries like India ( 65 - 75 percent ) . In developed countries, it is 14 percent according to recent WHO estimation.

In developing countries, prevalence of anaemia is higher. \& in India has 80 percent of maternal deaths due to it.(3) Inadequate dietary iron, foliate intake due to low vegetable 


\section{ORIGINAL ARTICLE}

consumption, perhaps low B12 intake and poor bioavailability of dietary iron from the fibre, phytate rich Indian diets are the major factors responsible for high prevalence of anaemia. Increased requirement of iron during growth and pregnancy and chronic blood loss contribute to higher prevalence in specific groups. In India, anaemia is directly or indirectly responsible for 40 per cent of maternal deaths, which comprises of 80 percent of villages, where majority of people live, and are ignorant of the hemopoietic principles of the diet and ignorant of regular antenatal checkup, so the incidence is high,

Depending upon the level of hemoglobin (HGB) concentration anaemia is graded (Degree) into

1. Mild degree - HGB percentage is $8 \mathrm{gm} / \mathrm{dl}$ to $11 \mathrm{gm} / \mathrm{dl}$,

2. Moderate degree - HGB percentage is $6.5 \mathrm{gm} / \mathrm{dl}$ to $8.0 \mathrm{gm} / \mathrm{dl}$,

3. Severe degree - HGB percentage is $6.5 \mathrm{gm} / \mathrm{dl}$ and less. (6).

If severe degree of anemia in pregnancy is left undiagnosed and untreated, it leads to consequence like preterm labor, pre-eclampsia, cardiac failure, intrauterine death (IUD) and the risk of developing anemia in infancy, intrauterine growth retardation (IUGR) defective mental and psychomotor development in the infant. (7).

Survey and detection of anaemia in pregnancy is most important to prevent maternal and perinatal morbidity and mortality. Five major surveys (National Family Health Survey (NFHS) and District Level Household Survey (DLHS), Indian Council of Medical Research (ICMR) Micronutrient Survey and Micronutrient Survey conducted by National Nutrition Monitoring Bureau(NNMB) were undertaken to estimate prevalence of anemia in the country. All these showed that over 70 percent of preschool children were anemic. NNMB, DLHS and ICMR survey showed that over 70 percent of pregnant women and adolescent girls in the country were anaemic (Table- 1). Anaemia begins in childhood, worsens during adolescence in girls and gets aggravated during pregnancy. NFHS 2 and 3 reported lower prevalence of anaemia in women and pregnant women as compared to DLHS, NNMB and CMR Micronutrient surveys a teaching Hospital patient based study is helpful in assessing true nature of problem in the community. Prevalence studies help in assessing the magnitude of anaemia in pregnancy and aid in devising preventive measures. Bijapur in northern Karnataka is an underdeveloped district with a large agrarian population. This study was undertaken to estimate the burden of anaemia in pregnancy in this part of the country, compare the prevalence rates in different part of India and to understand the dynamics of anaemia in pregnancy.

MATERIAL AND METHODS: The study was carried out at Al- Ameen medical college and Hospital, Bijapur, Karnataka state from January 2012 to December 2012. Subjects includes for the study were pregnant women attending the inpatients and out patients for antenatal checkup. Only selected 100 pregnant women having hemoglobin less than $11 \mathrm{gm} \% \mathrm{dl}$ and regularly attended antenatal checkup at Al-Ameen medical college and Women's Hospital, Bijapur, District, Karnataka State, hemoglobin (HGB) less than $11 \mathrm{~g} / \mathrm{dl}$ selected for the study those attended antenatal checkup regularly from IST to IIIrd trimester, Along with detailed prescheduled and redesigned examination proforma of all the pregnant women, including obstetrics' clinical history includes age, gravida, and parity etc were included in the study. Permission was obtained from the Institution Ethics Committee. $2 \mathrm{ml}$ of pregnant Blood sample collected in a EDTA K3 bulb and samples are analyzed in Automated 


\section{ORIGINAL ARTICLE}

Hematology Analyzer Sysmex Kx 21 (Tran Asia) hematological parameters used in this study are hemoglobin, Hematocrit, Mean corpuscular volume, Mean corpuscular haemoglobin, Mean corpuscular haemoglobin concentration, red cell distribution width in coefficient variation and peripheral smear study was done to know the morphological types of anaemia. All those pregnant women with clinical infections, chronic diseases haemo-globinopathies, multiple pregnancies and not attended regular antenatal checkup were excluded from the study.

RESULTS: Present series of study includes 100 cases of anaemia in pregnancy,

The age wise distribution of anaemic cases, prevalence is high in the age group of 21-25 yrs is $41(41.0 \%)$ and prevalence is lowest in the age group of 15- 20 yrs is 11 (11.0) - (Table 1).

Degrees of anaemia according to age in pregnancy are,

Mild degree of anemia in age group of 21 - 25 yrs is seen in 8 cases, moderate degree of anemia in the age group of 21- 25 yrs in 30 cases and severe degree of anemia is seen in $31-35$ yrs of age -6 cases (Table-2).

\section{Gravida and Degree of anaemia (Table -3)}

The degree of anemia is seen to increase with the gravida of the patient Maximum number of mild degree anaemia is seen in $4^{\text {th }}$ gravida of pregnancy. ( 9 cases); Maximum number of moderate degree of anemia is seen in $4^{\text {th }}$ gravida of pregnancy. (29 cases); Maximum number of severe degree of anemia is seen in $2^{\text {nd }}$ and $4^{\text {th }}$ gravida of pregnancy. (4 cases)

\section{Parity and degree of anemia (Table - 4)}

The degree of anemia is seen to increase with the parity of the patient. Maximum number of mild degree anaemia is seen in para 3 of pregnancy. (07 cases); Maximum number of moderate degree of anemia is seen in para 2 \& Para 3 of pregnancy. (29 cases); Maximum number of severe degree of anemia is seen in para -3 of pregnancy. ( 5 cases)

Trimester and Anemia (table -5)

Mild degree of anemia is seen high in $1^{\text {st }}$ Trimester and low in $3^{\text {rd }}$ Trimester; moderate degree of anemia is seen high in $3^{\text {rd }}$ Trimester and low in $1^{\text {st }}$ trimester; severe degree of anemia is seen high in $3^{\text {rd }}$ trimester and low in $1^{\text {st }}$ trimester

Socioeconomic status play a important role in development of anaemia in pregnancy specially in developing counties like India, due to ignorant of hemopoietic principles of diet and poverty and ignorance. (Table - 6)

Low income group patients shows more cases of moderate degree anaemia cases are 40 and Middle income group patients' shows more cases of moderate degree of anemia are 21 High income group patients show more cases of moderate degree of anemia are 09. 
Different types of anemia's seen in pregnancy are - (Table - 7)

Microcytic hypochromic anemia - 70 cases (70 .0\%)

Macrocytic normochromic anemia - 17 cases (17\%),

Dimorphic anemia - 13 cases (13\%)

Table-1: Age wise distribution of 100 anaemic cases in pregnancy

\begin{tabular}{|c|c|c|c|c|}
\hline AGE & $\begin{array}{c}15-20 \\
\text { yrs }\end{array}$ & $\begin{array}{c}\mathbf{2 1 - 2 5} \\
\text { yrs }\end{array}$ & $\begin{array}{c}\mathbf{2 6 - 3 0} \\
\text { yrs }\end{array}$ & $\begin{array}{c}\mathbf{3 1 - 3 5} \\
\text { yrs }\end{array}$ \\
\hline No. & 11 & 41 & 33 & 15 \\
$\%$ & 11 & 41 & 33 & 15 \\
\hline
\end{tabular}

Table-2: Degree of anaemia with age.

$\mathrm{X}^{2}=5.2, \mathrm{P}<0.05, \mathrm{df}=2$

\begin{tabular}{|c|c|c|c|c|c|}
\hline Degree of anaemia & $\mathbf{1 5 - 2 0}$ yrs & $\begin{array}{c}\mathbf{2 1 - 2 5} \\
\text { yrs }\end{array}$ & $\begin{array}{c}\mathbf{2 6 - 3 0} \\
\text { yrs }\end{array}$ & $\begin{array}{c}\mathbf{3 1 - 3 5} \\
\text { yrs }\end{array}$ & Total \\
\hline Mild & 03 & 08 & 06 & 01 & 18 \\
\hline Moderate & 12 & 30 & 24 & 04 & 70 \\
\hline Severe & 00 & 03 & 03 & 06 & 12 \\
\hline
\end{tabular}

Table-3: Gravida and Degree of anaemia.

\begin{tabular}{|c|c|c|c|c|c|}
\hline Degree of Anaemia & G1 & G2 & G3 & G4 & Total \\
\hline Mild & 01 & 04 & 04 & 09 & 18 \\
\hline Moderate & 18 & 06 & 17 & 29 & 70 \\
\hline Severe & 02 & 04 & 02 & 04 & 12 \\
\hline
\end{tabular}

$\mathrm{X}^{2}=1.32, \mathrm{P}<0.05, \mathrm{df}=2$

Table-4: Parity and grade of anaemia.

\begin{tabular}{|c|c|c|c|c|}
\hline \multirow{2}{*}{ Grade of Anaemia } & \multicolumn{4}{|c|}{ Parity (P) } \\
\cline { 2 - 5 } & P0 & P1 & P2 & P3 \\
\hline Mild & 04 & 03 & 04 & 07 \\
\hline Moderate & 09 & 10 & 29 & 22 \\
\hline Severe & 02 & 03 & 02 & 05 \\
\hline
\end{tabular}

$\mathrm{X}^{2}=1.39, \mathrm{P}<0.05, \mathrm{~d} \mathrm{f}=2$. 


\section{ORIGINAL ARTICLE}

Table- 5: Trimester and anemia.

\begin{tabular}{|c|c|c|c|c|}
\hline $\begin{array}{c}\text { Degree of } \\
\text { Anaemia }\end{array}$ & I st & II nd & III rd & Total \\
\hline Mild & 11 & 04 & 03 & 18 \\
\hline Moderate & 13 & 25 & 32 & 70 \\
\hline Severe & 00 & 03 & 09 & 12 \\
\hline
\end{tabular}

Table- 6: Socioeconomic status and anaemia.

\begin{tabular}{|c|c|c|cc|}
\hline $\begin{array}{c}\text { Degree of } \\
\text { Anaemia }\end{array}$ & LIG & MIG & HIG & Total \\
\hline Mild & 10 & 05 & 03 & 18 \\
\hline Moderate & 40 & 21 & 09 & 70 \\
\hline Severe & 07 & 03 & 02 & 12 \\
\hline
\end{tabular}

LIG=Low Income Group.

MIG=Middle Income Group.

HIG = High Income Group

Table-7: Type of anaemia according to peripheral smear.

\begin{tabular}{|c|c|c|}
\hline Types & $\begin{array}{c}\text { No. of } \\
\text { Cases }\end{array}$ & $\mathbf{\%}$ \\
\hline Microcytic Hypochromic & 70 & $70 \%$ \\
\hline Macrocytic Normochromic & 17 & $17 \%$ \\
\hline Dimorphic anemia & 13 & $13 \%$ \\
\hline
\end{tabular}

DISCUSSION: Prevalence of anaemia is highest in India, and also throughout the world. In our hospital based population study, Anaemia is one of the most common medical conditions met during pregnancy. Microcytic hypochromic anaemia accounts for $70 \%$ and dimorphic anaemia $17 \%$ and megaloblastic anaemia accounts for $13 \%$ of cases. Since majority of women enter pregnancy with partially or completely depleted iron reserves, the prophylactic use of iron has been advocated by most workers. Iron therapy not only prevents iron deficiency, but also reduces the incidence of dimorphic anaemia. Iron deficiency is more predominant due to poor intake and poor availability of iron in food. Anaemia would be more common if patient is infested by parasites like malaria, hookworm and who suffers from gastrointestinal diseases, like chronic dysentery and diarrhea. As a result of physiological anaemia in pregnancy there is disparity in red cell mass and plasma volume. The routine investigations of anaemia are helpful to detect the anaemia type. Although a reduction of circulating haemoglobin is relatively late development of iron deficiency anaemia, the measurement of haemoglobin RBC, indices hematocrit and RDW-CV, are done by Automated hematology analyzer SysmexKx 21 is the simple, noninvasive and time saving and accurate, reproducible $(6,8,7)$. In all these series maximum number of cases were observed between the age group of 21 to 30 years. Trimester wise distribution of present study $34 \%$ cases were in Ist trimester, $32 \%$ of cases were in II 


\section{ORIGINAL ARTICLE}

nd Trimester and 44\% of cases were in the III rd Trimester, L.P. Dutta et al $1972(9,10)$. Satyanarayan M and Ali, 1985, found in his series 115 of cases were in II nd trimester and $89 \%$ of cases were in III rd trimester. C. Sexena et al1970: found in his series 19\% cases were in IInd trimester and 24 cases in III trimester (11), Comparing various authors study it is seen that majority of cases are found in III trimester. Gravida and anemia of present study nearly correlated to the Menendez C., 2000. (12). Sexena C. et al 1970; (13)." found 9.80\% cases was primi gravida and $60.78 \%$ cases was in gravida two to gravida three and $29.41 \%$ were grandmultipara. Sidhu G.S1988; (14) had $15.15 \%$ of primi gravida, $57.57 \%$ cases was gravida two to gravida three and $27.27 \%$ were grand multi. M. Satyanarayana et al, 1985 had 16\% primi gravida, 74\% were grand multi (15); had $16 \%$ cases was primi gravida, $74 \%$ of cases were in multi gravida and $10 \%$ cases were in grand multi.

In present it is found that keeping haemoglobin standard as $11 \mathrm{gm} / \mathrm{dl}$, found $18 \%$ of cases mild anaemia, $70 \%$ cases moderate and $12 \%$ was severe degree anaemia. Rajaratnam et al 2000; in his study Keeping standard haemoglobin as $11 \mathrm{gm} / \mathrm{dl}$ found $30.2 \%$ cases was mild anaemia, 35.8\% cases of moderate anaemia and 3.3\% cases of severe anaemia L.P. Dutta et al $(16,17)$; Keeping haemoglobin standard as $10 \mathrm{gm} / \mathrm{dl}$ found $20 \%$ of cases was mild anaemia, $30 \%$ of case was moderate and $42 \%$ of case was severe anaemia. It is obvious that in our patients the moderate and mild anaemia are maximum. Hence our study closely correlated with the study of Rajaratnam and Dutta L.P (17). In our present study, morphological types of anemia's in pregnancy were $70 \%$ of cases are microcytic hypochromic, $17 \%$ of cases were dimorphic anaemia and $13 \%$ of cases were megaloblastic anaemia. Raja Ratnam et al in his study he was found 845 cases of iron deficiency, 9\% cases of dimorphic anaemia 6\% cases were of megaloblastic anaemia. Dutta L.P. in his series found $76 \%$ of cases were iron deficiency, $14 \%$ of cases were dimorphic anaemia and $10 \%$ of cases were megaloblastic anaemia. So present hospital based study correlates with Raja Ratnam and Dutta L.P. $(16,17,18)$;

Preventive Measures of anemia: The various methods that are used to health education reduce the prevalence of anaemia during pregnancy (25). Health education is an important approach to increase awareness about anaemia in terms of exposure, risk factors, essential nutrition ingredients and the importance of iron supplementations. The health education is the responsibility of both women and healthcare providers (18). Health education is an essential factor and most common approach of great importance to reduce the prevalence of anaemia (2519S). Many women are not aware about their medical condition or the risk factors which is threat their life .Women play an important role in the family as the main health providers and very eager to find information regarding their health condition

Health education and preventive measure during pregnancy are crucial factor for both mother and their offspring health because each pregnant woman is at risk during pregnancy. Therefore, it is important to raise the level of awareness for all women specially women in the reproductive age through effective and well organized health education and campaigns, with focus on major health problems that might occur during pregnancy, in particular anaemia. Therefore, for the success and effectiveness of health education, it is better that the information is provided to women in groups, where they feel more comfortable, satisfied and interact to benefit from each other (23). 


\section{ORIGINAL ARTICLE}

Ethical committee clearance: The ethical committee clearance obtained from ethical committee of Al-Ameen Medical College, Bijapur.

\section{Recent Diagnostic methods:}

1. Serum Vitamin B12, Serum iron, Folic Acid, Total Iron Binding capacity,

2. Bone Marrow Examination, with special stains like Pearl's stain

3. Serological methods to predict the risk of HDN - Rh Antibodies; cold antibodies, PNH.

Although the methodology and serological testing have improved significantly, the ability of these tests to predict hemolytic disease of Newborn with certainty is far from perfect.

Such tests will always be considered (20)

Supplementary to obstetric monitoring: Routine Hemoglobin, ABO and $\mathrm{Rh} \mathrm{D}$ group, of all pregnant women should be determined as early as possible during each pregnancy, when they first attend for antenatal care.-

Blood groups antibody detection is to be considered when there is history of miscarriage.

Mother's serum should also be screened for any IgG antibodies to red cells, which can cause HD (21).

Quantification of antibodies: Once detected, the ability of an antibody to cause HDN is generally estimated by antibody quantification. Serial testing (at least once monthly) Provides useful information regarding the antibody trend through pregnancy. Rising levels may be indicative of HDN developing in the fetus, but not confirm the blood group of the fetus or the severity of hemolysis. Detection of fetal red cells in the maternal circulation, one of the earliest methods used to detect fetal red cells in maternal circulation was described by Kleihauer and Betke. The rosette test, There are many different methods in use for the detection of a larger than usual fetal to maternal hemorrhage. The enzyme-linked antiglobulin test. The test with fluoresce in-labeled antibodies, and for evaluation of the feto-maternal hemorrhage. Nowadays Functional cellular assays tests are used. Serological tests indicate whether an antibody is of clinical importance, but they do not predict its functional activity. Antibody-dependent cellular cytotoxicity- (ADCC) assay and the chemiluminescence test Using maternal plasma and real time PCR with its automation opened the possibility for examining a large number of samples. (22).

Obstetric monitoring: Amniocentesis: The level of bilirubin in the amniotic fluid gives an indirect assessment of the severity of fetal heterolysis. -Ultrasound examination Ultra-sonography is a noninvasive method that can be used to study fetal hemodynamics and show physical indications of developing anaemia before hydrops develops.

Fetal blood sampling: The best method to assess s hemolytic disease is the direct determination of fetal haemoglobin, (HbF) hematocrit, and blood groups by testing a blood sample (23).

Prevention and management of chronic anaemia in pregnancy: The prevention of chronic anaemia needs blood transfusion during pregnancy can be reduced by adequate antenatal care. 


\section{ORIGINAL ARTICLE}

Blood Transfusion: It is important to remember that transfusion does not treat the cause of anaemia or correct the non-hematological effects of iron-deficiency. The decision to transfuse blood must not be based on patient's haemoglobin concentration alone, but also on her clinical needs, including stage of pregnancy and clinical conditions. Where iron and foliate deficiency is common, in countries prophylactic administration of hematinic is strongly indicated.

The optimum daily doses to prevent anaemia in pregnant women are: $500 \mu \mathrm{g}$ folate, $120 \mathrm{mg}$ elemental iron: e.g. $200 \mathrm{mg}$ tablet of ferrous sulfate (24).

CONCLUSION: In our study of hospital based population of the prevalence of anaemia in pregnancy, the incidence of anaemia is in pregnancy is high. Moderate to severe degree anaemia common in IIIrd trimester. In 100 cases studied for anaemia in pregnancy, 70\% cases is microcytic hypochromic category.17\% cases of megaloblastic anaemia seen in third trimester of pregnancy, $13 \%$ of dimorphic anaemia is seen in this study. Anaemia is common in age group between 21 -30 yrs, due to repeated and short interval pregnancies. SysmexKx 21 automated hematology analyzer is simple and reproducible instrument used during this study period the results are accurate and tally with conventional methods and P S.

During Pregnancy there is need for mandatory regular supply of Iron, folic acid and vitamin. B12 because prevalence of anaemia in pregnancy is very high in teaching based hospital population. To reduce the prevalence of anaemia and reduce magnitude of its problem, it is essential that antenatal health workers and qualified dietician should reinforce the daily iron, folic acid and vitamin B12 tablets to adolescent and pregnant women from 4th month onwards till 3-6 months of post-partum period along with correction of other nutritional deficiencies and timely intervention for reducing the burden of related diseases and food aid program towards illiterate domestic pregnant women is advised to reduce the prevalence and consequences of anaemia, not only this the antenatal care workers in teaching institute must be detected early and treat the anemia to reduce the prevalence rate of anemia in pregnancy. We hypothesize that this may be due to better awareness of risk of anaemia in pregnancy in city dwellers. The patients attending our hospital represent cross section of Bijapur population with mix of poor and rich as well as urban and rural. Therefore this study highlights the prevalence of anemia in pregnancy in this part of the country and shall provide reference to future studies on anaemia.

\section{REFERENCES:}

1. Firkin Frank, Chester man Colin et al 1989: “degruchy's clinical haematology in medical practice" 5th Ed., oxford university press; 29 pp.

2. Krishna Menon M. k., Devi P.K Bhaskerroa K., 1989: "Postgraduate obstetrics and gynecology", 4th Ed. Orient Longman, $61 \mathrm{pp}$.

3. K Kalaivani, Prevalence and Consequences of anaemia in pregnancy. Indian J Med Res 130, November 2009, 627-633 pp.

4. Anamika awasthi, R. Thakur, et al. "Maternal" Perinatal out come in cases of moderate and severe anaemia" j. of Obst and Gyn of India. 51 (6)0: 2001, 62-65 pp.

5. Bruno de Benoist et al; World wise prevalence of anaemia 1993-2005; WHO.

6. Neena Sharma "Iron deficiency anaemia in women: Role of carbonyl Iron". Obs \& Gynaec Today: VI (7): 2001, 399-403pp. 


\section{ORIGINAL ARTICLE}

7. Indian J Med Res 130, November 2009, 627-633 pp. Prevalence \& Consequences of anaemia in pregnancy K. Kalaivani.

8. Indian J Med Res 130, November 2009, 627-633pp Prevalence \& consequences of anaemia in pregnancy K. Kalaivani.

9. Anamikaawasthi, R. Thakur, et al. "Maternal" Perinatal out come in cases of moderate and severe anaemia" J of Obst and Gyn of India. 51 (6)0: 2001, 62-65 pp.

10. Pinto M, Rosario, 1970: “Toxemia of pregnancy”, J Obt and Gynecology' 154-158pp.

11. Dutta L.P., Nirupama Dutta, 1972; J obst. And Gyn. 20; (4; 26.), M Satyanarayana et al (8. 80 Satyanarayan M and Alli, 1985 "Anaemia complicating pregnancy", J Obt and Gyn of India 35(11); 335-338pp.

12. Dutta L.P, Nirupama Dutta, 1972; J Obst and Gyn. 20; (4; 26.), M Satyanarayana et al (10 .80Satyanarayan M and Alli, 1985 "Anaemia complicating pregnancy", J. Obt. And Gyn. Of India 35(11); 335-33 pp.

13. Sexena C. et al 1970: "Toxemia of pregnancy", J Obt and Gyn of India, vol20, 166-169 pp.

14. Menendez C., Fleming A.F., Alonso P.L., 2000: "Malaria related anaemia", parasitol Today, 16 (11; 469-476.)), C. Sexsena et al (13. 81 Sexena C. et al 1970: "Toxemia of pregnancy", J Bot and Gyn of India, Vol 20, 166-169 pp.

15. Sidhu G.S., Sood S.K., Ramalinga Swami V. 1988; "anaemia of pregnancy in northern India Delhi Area”, Indian journal of med. Res., 55 (6), 549-557 pp.

16. Satyanarayan M and Alli, 1985 "Anaemia complicating pregnancy", J Obt and Gyn. Of India 35(11); 335-338 pp.

17. Rajaratnam et al 2000; "Maternal anaemia: A persistent problem in rural Tamilnadu", Nutr. Med. J India 13 95; 242-245 pp.

18. Dutta L.P., Nirupama Dutta, 1972; J. Obst. And Gyn. 20; (4); 26 pp.

19. Dutta L.P., Nirupama Dutta, 1972; J. Obst and Gyn. 20; (4); 26 pp. 


\section{ORIGINAL ARTICLE}

\section{AUTHORS:}

1. Mohammed Arifulla K.

2. A.M. Patil

3. B. B. Sajjanar,

4. Sayeed M. Yendigeri

5. Vivek P. Honakeri

6. Nasheen Fathima

7. Nida Nausheen

\section{PARTICULARS OF CONTRIBUTORS:}

1. Assistant Professor, Department of Pathology, Al-Ameen Medical College and Hospital, Bijapur.

2. Professor \& Head, Department of Pathology, Al-Ameen Medical College and Hospital, Bijapur.

3. Professor, Department of Pathology, AlAmeen Medical College and Hospital, Bijapur.

4. Associate Professor, Department of Pathology, Al-Ameen Medical College and Hospital, Bijapur.

5. Assistant Professor, Department of Pathology, Al-Ameen Medical College and Hospital, Bijapur.
6. Assistant Professor, Department of Pathology, Al-Ameen Medical College and Hospital, Bijapur.

7. Assistant Professor, Department of Pathology, Al-Ameen Medical College and Hospital, Bijapur.

\section{NAME ADRRESS EMAIL ID OF THE CORRESPONDING AUTHOR:}

Dr. Mohammed Arifulla. K, Assistant Professor, Department of Pathology, Al-Ameen Medical College, Bijapur. Email -arif10mohdk@gmail.com

Date of Submission: 18/06/2013. Date of Peer Review: 18/06/2013. Date of Acceptance: 23/08/2013. Date of Publishing: 27/08/2013 